\title{
LAS MÚSICAS EN LA FORMACIÓN DE LOS MAESTROS Y MAESTRAS \\ EN EDUCACIÓN PRIMARIA. \\ ¿PUEDE LA MÚSICA CONCIENCIAR EN LA DIVERSIDAD DE GÉNERO?
}

\author{
MUSIC IN THE TRAINING OF TEACHERS IN PRIMARY EDUCATION. \\ CAN MUSIC RAISE AWARENESS OF GENDER DIVERSITY?
}

Ana Vernia Carrasco

Universitat Jaume I

\section{RESUMEN}

La educación es una responsabilidad, independientemente de la asignatura o materia de la que se trate, pues adquirir conocimientos no siempre garantiza la educación, en cambio educar sí que supone transmitir conocimientos y valores. En los últimos tiempos estamos asistiendo, lamentablemente, a una grave situación respecto a la diversidad de género, a pesar que la sociedad ha avanzado mucho, determinados temas sexistas siguen presentes, también en la educación. La música, elemento que vive y convive en personas de todas las edades, formando parte de sus vidas, se convierte también en una manera de entender la vida, los valores o las emociones. En este sentido, opinamos que la educación debe atender este factor como clave fundamental dentro de la formación del maestro y maestra, pues de ello también dependerán las canciones y músicas que se enseñan y aprenden en las aulas. Todavía seguimos escuchando repertorios alejados de compositoras, eclipsando así talentos, que siempre han estado, y que todavía muchos están por descubrir. Por una parte, proponemos una breve revisión de las guías docentes de la formación de maestros y maestras, y por otra parte, una propuesta para dotar de herramientas compositivas en el aula, desde unas recomendaciones en el repertorio. Cómo escogemos músicas y canciones es importante, y también tener recursos que permitan a futuros y futuras docentes poder componer pequeñas canciones, para trabajar en las aulas. Desde antaño, las canciones han sido utilizadas para diferentes aprendizajes, numéricos, poéticos, geográficos, o de género. La confección de un cancionero fue ya una propuesta que permitía asimilar contenidos teóricos musicales, ahora deseamos ir más allá y prestar atención a las letras, a su significado, a los roles que pueden marcar. Esto nos lleva a una interdisciplinariedad, desde la necesidad de atender las diferentes competencias que se demandan en educación primaria, tanto desde el alumnado como del profesorado.

Palabras clave: formación, educación primaria, música, canciones. 


\begin{abstract}
Education is a responsibility, independently of the subject or subject matter, since acquiring knowledge does not always guarantee education. Instead, educating means transmitting knowledge and values. In recent times, we are witnessing, unfortunately, a serious situation regarding gender diversity. Despite the fact that society has advanced a lot, certain sexist themes are still present, also in education. Music, an element that lives and coexists with people of all ages, being part of their lives, also becomes a way of understanding life, values or emotions. In this sense, we believe that education should address this factor as a key in teacher training, since it will also depend on the songs and music that are taught and learned in the classroom. We are still listening to repertoires away from women composers, eclipsing talents, which have always been there, and still many are yet to be discovered. On the one hand, we propose a brief review of the teaching guides for the training of teachers, and, on the other hand, a proposal to provide compositional tools in the classroom, offering some recommendations for the repertoire. How we choose music and songs is important, and also, to have resources that allow future teachers to compose small songs, to work in the classroom. Since ancient times, songs have been used for different learning, numerical, poetic, geographical, or gender. The making of a songbook was already a proposal that allowed assimilating musical theoretical content, now we want to go further and pay attention to the lyrics, their meaning, the roles they can signal. This leads us to interdisciplinarity, from the need to attend the different competences that are demanded in primary education, both from the students and from the teaching body.
\end{abstract}

Keywords: formation, primary education, music, songs.

\title{
SUMARIO
}

1. Introducción. 2. Marco Teórico. 2.1. Coeducación en el futuro maestro de primaria. 2.2. Música y Coeducación. 3. Marco metodológico. 4. Propuesta didáctica. 4.1. Objetivos. 4.2. Acciones y actividades. 5. Análisis de los resultados. 6. Conclusiones. Referencias Bibliográficas

\section{Introducción}

La disciplina musical, como cualquier otra, también ha sido y sigue siendo portadora de ese sesgo de género, incluso la terminología utilizada en el Lenguaje Musical viene cargada con connotaciones sexistas tales como las cadencias femeninas, por ejemplo, o las terminaciones imperfectas de las frases femeninas (Loizaga, 2005).

Cuando se habla de arte, personajes como Miguel Ángel, Leonardo da Vinci, El Greco, Velázquez, por citar algunos, no escapan al conocimiento de una persona con un mínimo de cultura general. Todo el mundo ha oído hablar de ellos alguna vez. Pero, ¿̇podríamos dar 
con la misma facilidad un sólo ejemplo de alguna pintora o escultora (...) así introduce Vázquez (s.f.) su artículo sobre las mujeres y la música. Este autor cita a mujeres relevantes en la Historia de la Música, como Hildegard von Bingen, Leonor de Aquitania o Lovise Adolpha Le Beau, entre otras, que han pasado desapercibidas (no para los melómanos o estudiosos de la música) pero sí para la gente de a pie e incluso para los docentes de música que pasan por encima de la propia historia sin llegar a conocerlas, para seguir ensañando la música desde los personajes masculinos (en su mayoría).

Martínez Cano (2017) también recurre a hechos históricos, en este caso a la mitología griega, para poner en evidencia nuestros lamentos cuando vivimos oprimidas, silenciadas o violentadas, refiriéndose a Démeter o Perséfone, viviendo y sirviendo a quien nos humilla, nos ignora o simplemente nos humilla. Como el lenguaje musical, esta autora también destaca el lenguaje masculino, cuando se refiere a que nuestro mundo está hecho para palabras masculinas. Además, como las mujeres no ocupan espacios destacados y relevantes en los contextos sociales, es más difícil poder acceder a nuevos contextos, así como controlarlos o visibilizarlos.

También los instrumentos musicales se relacionan con el género, así, instrumentos de viento metal tales como la trompeta, trombón, tuba, trompa o instrumentos de percusión han estado vinculados al género masculino, aunque no ocurre de esta forma en la actualidad, donde, cada vez más, podemos ver mujeres entre estos instrumentos musicales. De la misma forma ocurre con la docencia en el ámbito de la formación en maestros y maestra de grado en infantil y primaria, donde el grueso de estas titulaciones está formado en su mayor parte por el género femenino.

En palabras de Valdebenito (2013), las cuestiones de género todavía no están resueltas tampoco en el ámbito educativo, considerando que los cambios que se puedan generar en educación deben contemplarse desde una perspectiva dual, equilibrando las aportaciones masculinas y femeninas, por ello entiende que debe empezarse por proporcionar a los estudiantes, desde la educación musical, una visión inclusiva de género, tanto a través de los diseños curriculares como de las prácticas educativas.

Hernández Romero (2011) nos dice que ya en el siglo XIX las asignaturas asociadas al sexo femenino eran solfeo, piano, canto y arpa y excepcionalmente el violín, aunque también podían asistir a las clases de composición. Esta autora destaca que en el reglamento del Real Conservatorio Superior de Madrid las alumnas sólo podían estudiar de historia y literatura del arte dramático y la música, canto, declamación, lengua italiana, solfeo general y preparatorio para canto, piano, arpa, acompañamiento elemental y superior. En cuanto a profesoras con plaza estable fueron diez, en la especialidad de canto y piano, frente a un número muy superior de hombres. 
En nuestra aula de futuros maestros y maestras de educación primaria y en la asignatura de Didáctica de la Expresión Musical, detectamos, por una parte, un menor número de chicos en clase respecto a un alto porcentaje de chicas en esta formación, $y$, por otra parte, una serie de actividades que son preferidas por ellos y otras por ellas:

\begin{tabular}{|l|c|c|}
\hline \multicolumn{1}{|c|}{ Actividades } & $\begin{array}{c}\text { Actividades preferidas } \\
\text { por los chicos }\end{array}$ & $\begin{array}{c}\text { Actividades preferidas } \\
\text { por las chicas }\end{array}$ \\
\hline Interpretar con voz & & $\mathrm{X}$ \\
\hline Interpretar con instrumentos & $\mathrm{X}$ & $\mathrm{X}$ \\
\hline Expresión corporal & $\mathrm{X}$ & \\
\hline Improvisar con el cuerpo & $\mathrm{X}$ & \\
\hline Percusión corporal & $\mathrm{X}$ & $\mathrm{X}$ \\
\hline Seguir el ritmo con el cuerpo & & $\mathrm{X}$ \\
\hline Seguir la melodía con el cuerpo & $\mathrm{X}$ & \\
\hline Dirigir al grupo & & \\
\hline Juegos con pañuelos, pelotas... & & \\
\hline
\end{tabular}

Figura 1: Actividades preferidas según género

Fuente: Elaboración propia

La figura anterior nos da una idea del tipo de alumnado que hoy tenemos en nuestras aulas, y que serán los futuros maestros y maestras del ya bastante entrado siglo XXI.

\section{Marco teórico}

En opinión de Díaz Mohedo (2005), palabras que compartimos, los y las docentes deben concienciarse de la relevancia de sus acciones a través de su práctica entendiendo el importante papel que desarrollan en temas de socialización y contribuyendo a las identidades de género, por ello deben ser conscientes, por ejemplo, en la elección y selección de materiales, en este caso en la clase de música, para no caer en lo que históricamente se ha venido incidiendo, asociando a cada género un tipo de rol. Esta autora insiste en que el profesorado de música debe preguntarse qué opciones ideológicas y de valor está legitimando en la práctica y cuestionarse si son las más adecuadas desde el punto de vista educativo. Además, el o la docente debe, sino es consciente ya, de conocer y no desmerecer la contribución de la mujer al ámbito musical. 
En el trabajo de Martí (1999) se destaca la superficial conciencia entre música y algunos problemas derivados de la sociedad como las cuestiones de género, aludiendo a que el gran poder de la música como herramienta para culturizar e ideologizar estriba, en general, en no ser capaz de ver a la música más allá del mero entretenimiento. Su artículo se inicia con diferentes cuestiones, algunas relacionadas con las opciones que la mujer escoge en el terreno profesional de la música. La música, como bien dice este autor, a través de su perfil comunicativo, incide en la construcción social de la realidad, pues a través de ella se puede contribuir a las estructuras sexistas de la sociedad o a la diferencia de clases.

Pero la realidad, según Ramos (2010), es que no hay relación directa entre el porcentaje de compositoras y el nivel de desarrollo económico o de políticas dirigidas a potenciar al género femenino, y esto se da cuando ya la mujer puede acceder a instituciones de enseñanza y más aún cuando la UNESCO recomienda promover políticas de igualdad.

Retomando el tema docente y la enseñanza musical, según (Loizaga, 2005) esta práctica ha sido cuantitativamente de dominio femenino, aunque en pocas ocasiones lideran el sector. Como dice esta autora, son las conductas emotivas y expresivas las que se asocian al género femenino, siendo su cometido, generalmente, el de maestra.

Por todo, es necesario que la coeducación en la formación del maestro, y en especial en la, lamentablemente escasa, formación musical esté contemplada en la tarea discente.

Para Iverson (2011), el aprendizaje basado en las diferencias de género puede ser una buena razón para motivar al estudiantado a tener el deseo de aprender. Su investigación, que se centró en estudiantes de música, tuvo entre otras conclusiones, que los y las docentes de música deberían estar bien dotados para abordar las diferencias de género a través de sus clases y programas de estudio, incidiendo en que pueden motivar al estudiantado de música, utilizando ésta para estimular la kinestesia e incorporando actuales géneros y estilos de música dentro del currículum.

\subsection{Coeducación en el futuro/a maestro/a de primaria.}

La coeducación es un concepto en movimiento y, aunque es muy amplio, generalmente se alude a la educación conjunta de hombres y mujeres. En este sentido, cabe distinguir sexo y género, recogiendo el primer concepto características físicas, genéticas o instintivas con las que nacemos y el segundo concepto hace referencia lo que marca la sociedad y cultura respecto a construcción que asigna rasgos de identidad, capacidades, aptitudes y roles distintos a las personas, estableciéndose así, el género masculino y el femenino AAVV (s.f.). 
La escuela mixta supuso un avance en la igualdad de sexo, pero como dice Subirats (2009) fue solo un paso intermedio hacia la coeducación para llevar adelante los proyectos de escuela coeducativa que suponga la eliminación de barreras de género y proporcionar a todos y todas los mismos recursos y oportunidades. Como bien dice esta autora, palabras que suscribimos, la educación escolar no supone solamente la adquisición de conocimientos, pues también se adquieren hábitos, valores o la construcción de personalidades, pues los conocimientos, como resalta Subirats, sólo son útiles cuando hay una estructura mental idónea. Cabe recordar, como dice Martín Seoane (2015), que la educación mixta significa lo mismo que coeducación, pues el principal objetivo de la coeducación es la superación del sexismo proporcionando desde la escuela el marco que permita un modelo diferente de relaciones sociales, transformando los conflictos de las escuelas mixtas en oportunidades para potenciar la igualdad y el respeto.

Entiendo la coeducación como una estrategia o método para la educación fundamentado en la igualdad de sexo, coeducar significa que no se puedan establecer dominios sobre nadie, en esta línea Blanco García (2007) nos dice que coeducar es educar a cada una y a cada uno según quién es, atendiendo a su diferencia, así, coeducar es también educar fuera del modelo dominante.

En este sentido, y pensando en la Educación Musical, debemos dejar que nuestro alumnado escoja tanto las actividades para llegar a la asimilación de contenidos como los instrumentos musicales o materiales que permitirán desarrollar sus capacidades y habilidades musicales.

\subsection{Música y Coeducación.}

No tenemos en el territorio español muchos estudios sobre música y coeducación, como dice Valdebenito (2013), los primeros estudios sobre música y género comenzaron desde la musicología en la década del 60' del siglo pasado. Ya en el siglo XXI seguimos tratando estos temas en la escuela, pero ¿̇cómo formamos a los/as futuros/as docentes en una materia como la música?, que ya muchas investigaciones nos detallan sus beneficios tanto en el terreno emocional como el social. Esta autora se plantea la posibilidad de incidir en el problema de separación de géneros desde la enseñanza musical y por ello considera dos posibles objetivos:

1. Incluir temas de género con una perspectiva inclusiva en los diseños curriculares en todos sus ámbitos (prescriptivos, de centro y de aula). 
2. Detener la segregación temática respecto del tema de género en la educación musical, de manera que no se siga un modelo que atienda a una perspectiva fragmentada, es decir, solamente femenina o solamente masculina, sino de integración.

Siguiendo con esta línea, consideramos que es fundamental, además, la formación desde la Universidad para los futuros maestros y futuras maestras, la selección de actividades y de instrumentos musicales, qué canciones les ofrecemos para las actividades en el aula y los roles que asumen para llevar a cabo las diferentes actividades de enseñanza-aprendizaje. Si nuestro estudiantado, futuros maestros y maestras, trabajan desde la coeducación en su formación, podrán aplicarlo en sus futuras aulas, no así, si seguimos impartiendo las clases tradicionales, con los métodos tradicionales y con las mismas herramientas que aprendimos nosotros/as.

La música desde tiempos remotos siempre ha tenido unas características y lazos que según en qué momento y de qué función se tratase, se entendía ligada a un género u otro, incluso el propio lenguaje musical, como ya avanzábamos, es sexista en su terminología y también entre sus instrumentos musicales o roles (director de orquesta, mánager o representante, técnico de sonido, etc.). Aunque el progreso en el marco de la formación musical ha evolucionado.

El estudio de Killian y Satrom (2011) muestra cómo los niños tienen la tendencia a escoger instrumentos musicales de viento metal y las niñas de viento madera. La experiencia se fundamentó en la presentación de diferentes instrumentos de viento madera y viento metal, interpretados por hombres y mujeres identificándose los participantes con su género.

En su investigación, Wrape, Dittloff y Callahan (2016) tomaron a 99 participantes de una banda de escuela secundaria, pudiendo comprobar que los estereotipos de géneroinstrumento se mantienen arraigadas y plantean un problema persistente frente a los docentes de música, resaltando que los miembros más jóvenes y sin experiencia en bandas están más abiertos. Estos autores señalan que hay estudios previos que señalan que los estereotipos de género están asociados con la elección del instrumento musical de los niños.

En opinión de Fernández-Carrión (2011) cuando cantamos, bailamos o escuchamos música asociada a ciertas actividades podemos estar transmitiendo ciertos roles diferenciando géneros, por ello la Educación Musical debe plantearse educar incidiendo en la igualdad. En nuestra opinión, la música es clave en la educación integral, pues la música está en la vida de todas las personas, tanto niños/as, adolescentes como adultos/as, nos acompaña en el trabajo y en el ocio, en la familia y en la salud, para la mejora de la calidad de vida, para la concentración, etc. por ello es necesario que se cuide tanto su enseñanza como su 
aprendizaje, pues si hay un/a docente detrás que parta de una formación adecuada a las necesidades y demandas actuales, la educación musical será eficiente en su cometido y por ello la universidad debe buscar un perfil que permita llevar a cabo esta labor.

La coeducación, hoy tema actual, es necesaria en el aula, pero como dice la autora anterior, los y las docentes somos conscientes de la relevancia de la música y cuáles son sus ámbitos desde los que se pueden identificar situaciones de desigualdad de género, atendiendo a que nuestra música tradicional está impregnada de valores sexistas y este repertorio forma parte de nuestra tradición cultural, no es necesario obviarlo pero sí tener en cuenta los mensajes que transmite y cuáles fueron sus contextos históricos.

No debemos olvidar los cambios sociales generados por la globalización y los restos que, según Burnard et al. (2008), ofrecen a los y las docentes de música dentro del aula, así como las oportunidades para investigar sobre la pedagogía crítica, valores y estrategias de enseñanza. A continuación, formulamos algunas de las preguntas que se plantean estos autores:

- ¿ ¿Nos preparamos con eficacia los/las futuros/as profesores/as de música? - ¿Qué podemos aprender de los/as maestros/as que han aprendido a hacer frente a los retos de la enseñanza musical?

- ¿ ¿Cómo fomentamos la motivación?

- ¿ ¿Cuáles son las funciones de los padres y madres?

- ¿Cómo son los estudiantes que están aprendiendo dentro y fuera de la escuela?

- ¿Cuál es la relación del profesorado con la política si los documentos que rigen no son aplicables o relevantes en la práctica?

Ante las anteriores preguntas y nuestra experiencia en las aulas de los futuros de docentes generalistas, nos planteamos la necesidad de desarrollar una propuesta didáctica que pueda responder a las necesidades e intereses de una sociedad cambiante, que no termina de cambiar, pues como bien señala Rusinek (en Burnard et al. 2008), a pesar de la prosperidad económica, hay un aumento de conflictos en la escuela como el absentismo, el bullying o el fracaso escolar, pues los/las docentes se consideran insuficientemente capacitados para hacer frente a los conflictos actuales ya que sus programas no los son. En este sentido podemos entender que los conflictos que se puedan generar por las diferencias de género estarían contemplados dentro de estas complicadas situaciones a los que todavía no se han puesto fin. 


\section{Marco metodológico}

En primer lugar, buscamos en nuestra guía docente, para conocer cuántas compositoras teníamos en el listado, sorprendentemente, de 43 propuestas de audiciones, 41 son de compositores, 2 figuran sin autor, por tanto 0 compositoras.

Podríamos denominar a nuestra metodología pre-observacional, ya que no hemos cumplido todavía con los parámetros que se deben incluir dentro de una metodología observacional, pues nos hemos limitado a observar y tomar nota de aquellas reacciones que nos parecían que estuviesen relacionadas con nuestras dudas/hipótesis. Así, tomamos notas de las actividades realizadas en clase dentro de la asignatura de didáctica de la expresión y recogimos datos de las Unidades didácticas que debían confeccionar con una selección de actividades dirigidas a adquirir las competencias musicales en el marco de la educación primaria, y que el estudiantado debía mostrar de manera activa, como si de un entorno real se tratase.

Insistiendo en que no hemos utilizado la metodología observacional, sí hemos tenido en cuenta que esta metodología estudia la conducta no verbal (movimientos corporales), la conducta espacial (la distancia espacial interpersonal en la comunicación), la conducta extralingüística (tono de voz, rapidez, secuencia en las intervenciones) y la conducta lingüística, comportamientos muy ligados a la expresión y educación musical.

Nuestra fase exploratoria puede resultar al/la experto/a excesivamente larga, pero las realidades en el aula nos permiten investigar en el aula con una velocidad determinada, así, utilizamos dos cursos académicos en el marco de la universidad y en una asignatura de 6 ECTS o créditos en los que actualmente se configuran las titulaciones universitarias. Durante el primer curso nos dimos cuenta que nuestra clase era mayoritariamente del género femenino y que el alumnado solía agruparse, para hacer trabajos grupales, por sexo, lo cual ya nos resultó característico dado que la sociedad ha cambiado o ha evolucionado respecto a la escuela mixta. Por otra parte, nos dimos cuenta de que las actividades de movimiento y expresión corporal y vocal estaban más cómodas en el ámbito femenino, mientras que la interpretación y/o dirección eran realizadas con mayor seguridad por el lado masculino.

Estas realidades que sucedían en el aula nos llevaron a plantearnos, ante la defensa de la Unidad Didáctica que utilizamos como parte de la evaluación en nuestra asignatura, si realmente habría una diferencia marcada entre actividades escogidas y género. 


\section{Propuesta didáctica}

Tomando como referencia las aportaciones de Subirats (1994), nuestra propuesta tendría en cuenta:

- La posición de las mujeres como profesionales de la enseñanza.

- El androcentrismo en la ciencia y sus efectos sobre la educación.

- El androcentrismo en el lenguaje.

- Los libros de texto y las lecturas infantiles.

- La interacción escolar.

El primer paso que debemos tomar, bajo nuestro punto de vista, es la selección de las pedagogías activas que comportan la expresión corporal, tales como Dalcroze y su aplicación en el aula. La expresión corporal, como ya hemos avanzado, es una estrategia que parece funcionar mejor en el género femenino, por tanto, es necesario desde el primer momento que el propio alumnado descubra los beneficios del cuerpo como herramienta de expresión, comunicación y aprendizaje musical, así como la voz. Cantar es uno de los caminos, a nuestro entender, más directo con la comprensión de diferentes elementos teórico musicales y, como ya mencionábamos, suponen diferencias de género en tanto técnica (muda de la voz) y repertorio. Por tanto, la selección de repertorio, como la creación o composición del mismo, puede significar cambios y modelos positivos en la inclusión y en mejora de diferencia de género, teniendo en cuenta siempre el contexto en el que se moverá el/la futuro/a docente y así lo debería entender cuando ejerza su profesión.

\subsection{Objetivos}

- Utilizar la música como herramienta de no exclusión educativa.

- Seleccionar repertorio musical que permita la igualdad de género.

- Componer canciones para fomentar la convivencia escolar entre ambos sexos.

\subsection{Acciones y actividades}

Para empezar a fomentar la educación musical atendiendo a la diferencia de género, la expresión corporal y vocal será el eje vertebrador de las actividades, atendiendo también a los contenidos teórico-musicales de la guía didáctica. 
- Actividades de expresión corporal:

- Seguir la música con el cuerpo como instrumento de comunicación.

- Diferenciar elementos agógicos y dinámicos.

- Diferenciar frases.

- Señalar el carácter musical.

- Actividades con la voz:

- Calentamiento vocal sin referencia tonal.

- Juegos vocales (onomatopeyas) explorando las posibilidades de la voz.

- Improvisaciones rítmico-vocales, explorando la creatividad.

tura y/o de memoria.

- Cantar (con notas musicales y letras de canciones) siguiendo parti-

- Actividades teórico-prácticas:

- Selección de canciones (nivel de Educación Primaria),

$\nabla$ Atendiendo a la letra (valores, emociones, tareas, etc.).

- Composición de canciones.

$\otimes$ Para motivar o potenciar determinadas actuaciones en clase y fuera de ella.

$\nabla$

\section{Análisis de los resultados}

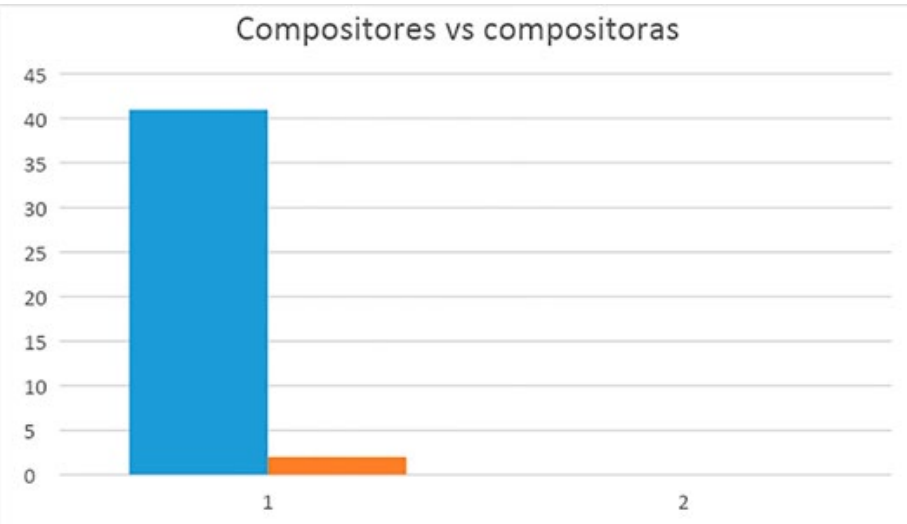

La serie 1 equivale al número de compositores (41). La serie 2 representa dos composiciones sin autor o autora (2). 


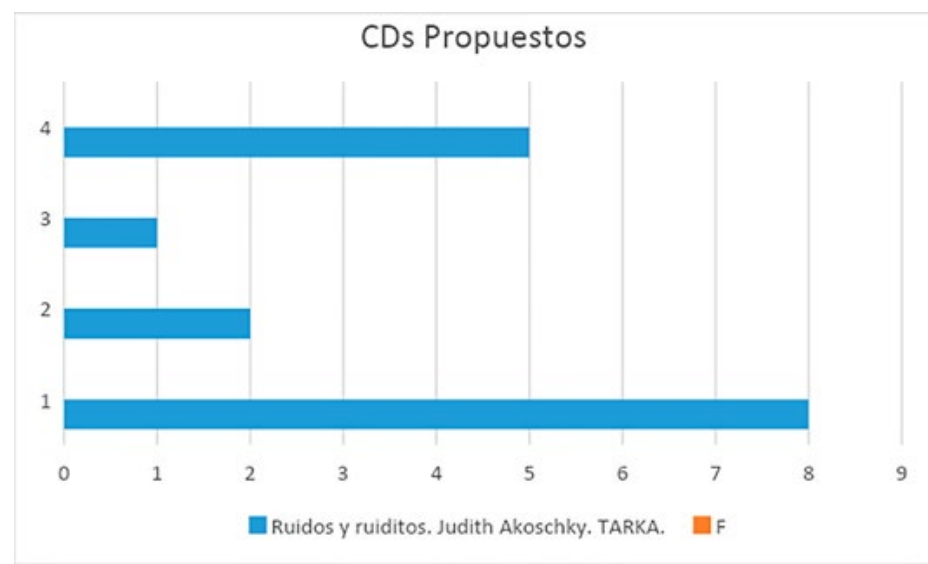

Figura 2: Cds propuestos

\section{Conclusiones}

Como dice Subirats (1994), aunque la educación es un fundamental para actuar sobre las desigualdades, no las puede hacer desaparecer. Por nuestra parte, la Educación Musical, además de los beneficios que ya conocemos por las diferentes investigaciones que van aflorando en los últimos años, puede ser una herramienta que eduque en la igualdad de género, que incida en la disminución de diferencias de sexo y que promueva el aprendizaje así como estrategias didácticas que eviten el riesgo de exclusión educativa.

Aunque no estamos ante una investigación al uso, pensamos que nuestra propuesta didáctica puede ser el inicio de una búsqueda para encontrar información que efectivamente nos dé la certeza de que efectivamente la música es un camino didáctico que requiere de una formación específica para desarrollar investigaciones que promuevan la mejora de la calidad educativa, y con ello entendemos también que no exista diferencia de género en las aulas. 


\section{Referencias}

Aavv (s.f.). Guía de Coeducación. Documento síntesis sobre educación para la igualdad de oportunidades entre hombres y mujeres. Red2Red Consultores S.L. Disponible en http://www.baiona.org/c/document_library/get_file?uvid=996cd4a5-9354-4ee2abc5-de5f1858095c\&groupld=10904 (Fecha de consulta: 2/8/18).

Anguera, María Teresa (2003). La observación. En C. Moreno Rosset (Ed.), Evaluación psicológica. Concepto, proceso y aplicación en las áreas del desarrollo y de la inteligencia (pp. 271-308). Madrid: SANZ y TORRES. [I.S.B.N. 84-96094-16-2]

Burnard, Pamela, Dillon, Steve, Rusinek, Gabriel. and Saether, Eva (2008) Inclusive pedagogies in music education: A comparative study of music teachers' perspectives from four countries. International Journal of Music Education 26(2), 109-126. http://ijm.sagepub.com/content/26/2/109. short?rss=1\&ssource=mfr Fecha de consulta: 5/6/18).

Blanco García, Nieves (2007) Coeducar es educar para la libertad. En Consejería de Educación. Andalucía educativa, 64. Sevilla: Junta de Andalucía. pp.24-27.

Díaz Mohedo, María Teresa (2005). La perspectiva de género en la formación del profesorado de música. REICE. Revista Iberoamericana sobre Calidad, Eficacia y Cambio en Educación, 3, 1, pp. 570-577 Disponible en http://www.ice.deusto.es/rinace/ reice/vol3n1_e/Diaz.pdf (Fecha de consulta: 2/7/18).

Fernández-CARRIÓn, Marta (2011). Música y género: estereotipos sexuales a través de la música. Red Educativa Musical. Disponible en http://recursostic.educacion.es/ artes/rem/web/index.php/es/curriculo-musical/item/360-m\%C3\%BAsica-yg\%C3\%A9nero-estereotipos-sexuales-a-trav\%C3\%A9s-de-la-m\%C3\%BAsica (Fecha de consulta: $2 / 7 / 18)$.

HeRnÁNDEZ Romero, Nieves (2011). Educación musical y proyección laboral de las mujeres en el siglo XIX: el Conservatorio de Música de Madrid. Trans. Revista Transcultural de Música, 15, pp. 1-41. Barcelona: Sociedad de Etnomusicología. Disponible en http:// www.redalyc.org/articulo.oa?id=82222646004 (Fecha de consulta: 5/7/18).

IVERSON, Brittany (2011). Music and gender: A qualitative study of motivational differences at the upper elementary level. Visions of Research in Music Education, 18. Disponible en http://wwwusr.rider.edu/vrme / (Fecha de consulta: 5/7/18).

Killan N, Janice, and Satrom, Shauna (2011). The Effect of Demonstrator Gender on Wind Instrument Preferences of Kindergarten, Third-Grade, and Fifth-Grade Students. Applications of Research in Music Education, 29, 13-19 
LoIzAGA, María (2005). Los Estudios de Género en la Educación Musical. Revisión crítica. Musiker. 14. Disponible en http://www.euskomedia.org/PDFAnlt/musiker/14/14159172.pdf (Fecha de consulta: 2/7/18).

Martínez Cano, Silvia (2017) Procesos de empoderamiento y liderazgo de las mujeres a través de la sororidad y la creatividad. Dossiers Feministes, 22, 2017, 49-72 - ISSN: 1139-1219 - DOI: http://dx.doi.org/10.6035/Dossiers.2017.22.4 (Fecha de consulta: $5 / 7 / 18)$.

Martín Seoane Gema (2015). ¿Educación mixta o diferenciada?: Coeducación. Boletín ECOS,30. FUHEM ECOSOCIAL. ISSN - 1989-8495. Disponible en https://www. fuhem.es/media/cdv/file/biblioteca/Boletin_ECOS/30/Coeducacion_G_MARTIN_ SEOANE.pdf (Fecha de consulta: 5/8/18).

MARTí, Josep (1999). Ser o hombre o ser mujer a través de la música: una encuesta a jóvenes de Barcelona. Horizontes Antropológicos, Porto Alegre, 5, 11, 29-51

Ramos, Pilar (2010). Luces y sombras en los estudios sobre las mujeres. y la música Revista Musical Chilena, 213, pp. 7-25. Disponible en http://www.scielo.cl/pdf/rmusic/ v64n213/art02.pdf (Fecha de consulta: 10/6/18).

SUBIRATS, Marina (2009). La escuela mixta żgarantía de coeducación? CEE Participación Educativa, 11, julio 2009, pp. 94-97. Disponible en http://www.mecd.gob.es/revistacee/pdf/n 1 1-subirats-martori.pdf (Fecha de consulta: 10/7/18).

SUBIRATS, Marina (1994). Conquistar la igualdad: la coeducación hoy. Revista lberoamericana de Educación, 6. Género y Educación. Disponible en 2016 de: http://rieoei.org/ oeivirt/rie06a02.htm (Fecha de consulta: 5/5/18).

VALDEBENITO, Lorena (2013). Educación Musical y Género: Una perspectiva inclusiva- desde el currículum de aula. Revista NEUMA. 6, 2. Universidad de Talca, pp. 58-66. Disponible en http://musica.utalca.cl/DOCS/neuma/2013-2/neuma\%20n\%C2\%BA2\%20 58-67.pdf (Fecha de consulta: 11/5/18).

VÁzQUEZ, Vicente (s.d.). Las mujeres y la música. Disponible en http://www.uv.es/ rsalvadp/ mujeres.htm (Fecha de consulta: 14/7/18).

Wrape, Elizabeth., DITTLOFF, Alexandra. and Callahan, Jennifer (2016). Gender and Musical Instrument Stereotypes in Middle School Children. Have Trends Changed? Applications of Research in Music Education, 34, 3 40-47. 